\title{
The Design of ETC System Based on RFID and Image Identification
}

\author{
Zhaoxia Tang \\ Faculty of Computer Engineering, Huaiyin Institute of Technology, Huai'an 223003, P.R.China
}

\begin{abstract}
This paper first analyzed the current situation of highway ETC system of domestic and international, then depth researched on some key technologies of RFID and image identification, established ETC system of city application, on the one hand, it can greatly improve vehicles management efficiency, prevent manual vulnerabilities, on the other hand, greatly promote the automation construction in the city.
\end{abstract}

Keywords-RFID; ETC (electronic toll collection), technologies of RFID; image identification

\section{INTRODUCTION}

According to National Bureau of Statistics released on February 22, 2012, the 2011 National Economic and Social Development Statistics Bulletin [1], National civil vehicle population has reached $105,780,000$, increased $16.4 \%$ than last year, private cars ownership was 78,720,000, increased $20.4 \%$. The civilian cars ownership was 49,620,000, increased $23.2 \%$. In this case, the use of ETC system (the vehicles do not need to stop for payment) has become an urgent need of social development. ETC system not only can greatly improve the traffic capacity; but also can reduce noise levels and emissions significantly, saving management costs, can make outstanding contributions to the improvement of the urban environment. In fact implementation and promotion of ETC system of city is more urgent than highway. Therefore, the introduction of ETC system of city, improve ETC range of applications, better to achieve the integration of the city.

\section{RESEARCH STATUS AND DEVELOPMENT OF THE ETC SYSTEM}

As more and more vehicles, manual and semi-automatic charging methods can not meet charge requirement, it will cause severe vehicle obstruction at the toll entrances, causing huge economic losses. In order to solve these problems, countries are active in the development and application of electronic toll collection system.

\section{A. Foreign Developments}

In the United States, electronic toll way has become the means of the high efficiency of US highway [2], the most famous networking running electronic toll collection system is the E-ZPASS system. The E-ZPASS system using dedicated lanes and mixed lane two modes, two modes have toll collectors on duty. Since then, ETC trading volume continued to grow.
In the Portugal, ViaVarde can be regarded as one of representative significance of networked electronic toll collection system in Europe [3], ETC system uses a combination of closed and open mode. The vehicle can pass more than the speed of $80 \mathrm{~km} / \mathrm{h}$. The fact has been proved, without ViaVarde system, the operating company will have to build more than 2,000 manual toll lanes to resolve congestion problems.

Japan takes contact CPU Calgary, two electronic tags and double ETC antenna program[4],lane set up high-speed bidirectional open railings, unattended, high security and lane capacity, it has key diffusion mechanism and electronic tags issued and outstanding system, but costs of lane system investment and electronic tags are high.

\section{B. Domestic Developments}

Beijing Capital Airport Expressway, the TianZhu toll station was installed the toll collection system in October 1996 [5], Equipment imported from the U.S. AMTECH, after several years, the system runs stably. Shanghai HongQiao International Airport used modular electronic toll collection system, the DSRC short-range communication with 5.8G microwave, and main, passive-compatible antenna. Guangdong Province has rapid development of Domestic highway, already using the combined charges of the two electronic tags and dual-interface CPU card technology solutions of ETC, establish customer service centers of 13 major cities.

At present, the electronic toll collection system in our country has more mature development and application. If extend to the city, can reduce the part of the operating costs of road management, reduce the financial burden; create a good traffic environment for public travel, and stimulate the economic development of the city. But at this stage electronic, toll collection system extend to the city, it has some problems, high charges cost, chaotic management, as well as vehicle low traffic rate etc.

In order to solve the above problems, ETC system is embedded RFID without changing the appearance of the original vehicle, when the vehicle enters the RFID reader operating range, Its own data information is transmitted to the reader, reader return to the background computer for processing after the receipt of information, completed once the identification of the relevant information on the vehicle, and automatically deduct the amount on the card, in order to complete toll transactions through the toll station without stopping. 


\section{RFID TECHNOLOGY}

\section{A. The RFID System}

A typical RFID system generally consists of three parts: RF card (RFID), reader and applications (including the connection line). System configuration as shown in Figure I.

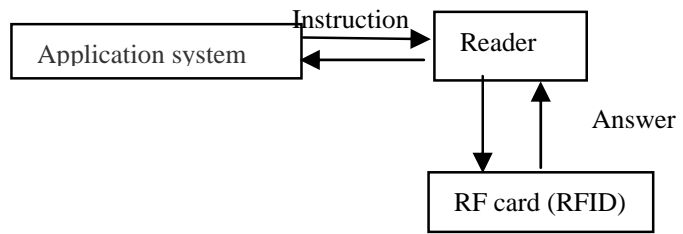

FIGURE I. RFID SYSTEM

In practical applications, electronic tag attached to the surface of the recognition object, electronic tags generally save the convention format of electronic data. The reader sends out a certain frequency of the RF signal through antenna, when the tag enters the magnetic field, the induced current to obtain energy, the encoded information is read and decoded by the reader, then sent to the computer, computer and network realize management functions such as collection, processing and remote transmission of object recognition information.

\section{B. RFID System Working Principle}

As shown in Figure II, Reader and RF card through wireless communication mode, they have wireless transceiver module and an antenna (induction coil). RF card can read and write real-time operations according to the reader send an instruction. The control module complete acceptance, decoding and executing the reader command, data security and other functions. Several modules integrated into RF card, Chip's peripheral simply connect the antenna. control module of reader have very strong processing function, in addition to complete the control of RF card task, Also need to achieve mutual authentication, data encryption, data validation, error alarm and communication with the computer and other functions.

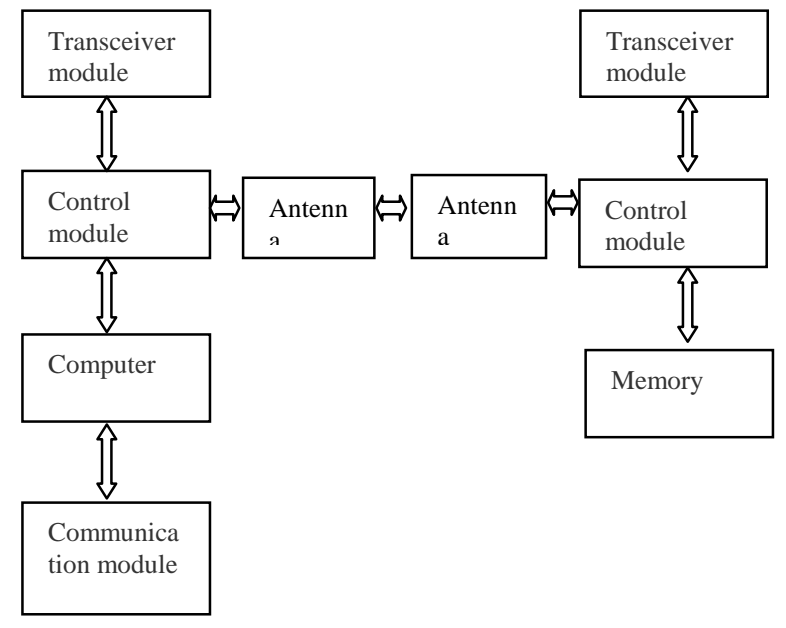

FIGURE II. RFID SYSTEM WORKING PRINCIPLE

\section{Anti Collision Technology}

According to the different working frequency of RFID, They put forward a lot of anti collision technology, in the high frequency (HF) band, anti collision technology generally adopts the classical ALOHA protocol, in the ultra high frequency (UHF) band, mainly using tree bifurcation algorithm to avoid collision [6]. These two anti collision algorithms belong to the TDMA method, are widely used. Due to the multi- vehicles of city's traffic, electronic tag is more and dense. In order to minimize the incidence of collision, by comparing binary search anti-collision technology and slotbased anti-collision technology, combine characteristics of ETC of city, by taking dynamically adjust the transmission slot time and packet size to reduce the incidence of the collision. The specific process is as follows.

First estimate tags, dynamic programming by forecast number of tags, draw the initial value of data packet and transmission slot time, the number of communication tags are obtained according to the actual situation, Derived optimized size of the data packets and the transmission slot, and for data transmission, until the completion of the communication process. If there are more tags in two time slot collision, then use request command to increase the available slot time.

By using the dynamic adjust data packet and transmission slot, when the amount of data and tag number is large, in this paper, the traditional slot-based anti-collision algorithm is optimized, the reader can read tags within a short period of time in the case of error rate.

\section{IV.IMAGE RECOGNITION TECHNOLOGY}

Image recognition technology is an important field of artificial intelligence. At present put forward two kinds of image recognition model to simulate human image recognition activity, template matching model and Prototype matching model.

\section{A. Vehicle Identification Technology}

The camera capture vehicle images to pre-processing, extraction of interesting region, license plate location and extraction, segmentation of license plate character, and extraction of the character image feature, then input the feature vector to the trained neural network or character template matching characters to identify.

\section{B. Vehicle Type Identification Technology}

At present, vehicle identification methods are mainly included infrared identification, identification with radar, ultrasonic recognition and image processing technology.

1. Infrared identification is divided into two categories: passive and active methods. it uses infrared detection principle of thermal object type to realize automatic recognition.

2. Radar identification use the Doppler principle, radar detector send microwave signals to the vehicle, then carry out vehicle identification by the reflected signal of vehicle size, shape and other factors. 
3. Ultrasonic recognition use ultrasonic object to detect.

4. Image processing technology identify vehicle model according to the different vehicle characteristic information. According to the national standards of various vehicle model, draw the characteristic parameters for the vehicle recognition, it is currently the most commonly used algorithm in vehicle identification.

\section{Vehicle Identification Method}

Samples of vehicle recognition generally have three types: front view, side view and top view, the most common is the side view. As shown in figure III, there are five kinds of vehicle types in the experimental samples, respectively, for the bus, truck, medium-bus, car and micro-bus. From the outside, the shape of the bus and truck is very clear and obvious different from other three vehicles. But between the car, medium-bus and micro-bus, physical appearance is not obvious, the characteristic of these three vehicles have some cross, therefore selected three feature sets [7]:

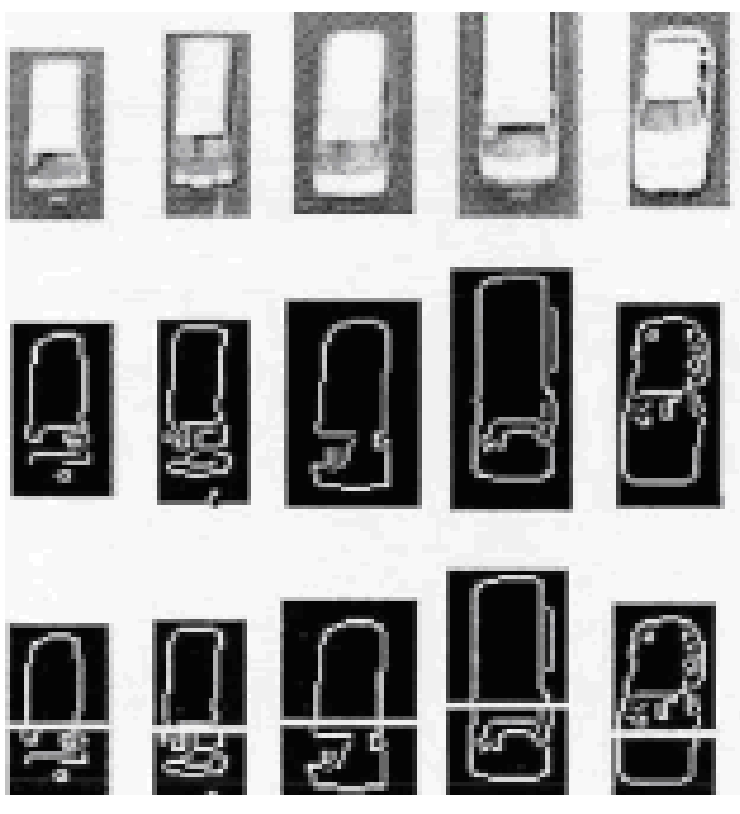

FIGURE III. FIVE KINDS OF VEHICLE TYPES

$$
\begin{aligned}
& \text { F1 }=\{\text { Length, Width, Area, Perimeter }\} \\
& \text { F2 }=\{\text { Edge, Area-Perimeter Ratio }\} \\
& \text { F3 }=\{\text { Length, Width }\}
\end{aligned}
$$

The marginal distribution characteristics of bus and microbus is similar, they are different from car in this feature. Edge density of bus's head significantly larger than bus's body, edge density of car's head was much smaller than car's body. Therefore, the edge density characteristics can be used to distinguish the medium-bus, micro-bus and car. The steps are as follows:

1) The first level classification: Through training classes' center as the clustering center, vehicle samples according to its feature vector and its class center to calculate Euclidean distance, where the smallest distance for the wooden category.
2) The second class classification: In the first level classification, bus and truck can be identified, the rest of the other vehicles include: medium bus, micro-bus and car. The center value of particle swarm method training as a threshold, throng the threshold, the car can be identified.

3) The third class classification: There are medium bus and micro-bus to distinguish. In obtaining the center, the Euclidean distance and the threshold can be carried out to class classification.

\section{DESIGN OF CITY ETC SYSTEM}

\section{A. Design of RFID and Reader}

Operating frequency of RFID tag has the low-frequency (LF 100-135kHz), high frequency (HF 13.56MHz), ultrahigh frequency (UHF 433MHz and 800/900 MHz) and microwave band (MF $2.45 \mathrm{GHz}$ and $5.8 \mathrm{GHz}$ ) [7]. RFID system of different bands are different of working principle, corresponding to the different read and write distance, as shown in Table 1.

TABLE I. DIFFERENT RFID TAGS OF OPERATING FREQUENCY

\begin{tabular}{|c|c|c|}
\hline Operating frequency & Advantage & Disadvantage \\
\hline $\begin{array}{c}\text { low-frequency } \\
\text { (LF) }\end{array}$ & $\begin{array}{c}\text { The technology is simple } \\
\text { and reliable }\end{array}$ & $\begin{array}{c}\text { Communication speed is } \\
\text { low, distance is short }\end{array}$ \\
\hline $\begin{array}{c}\text { high frequency } \\
\text { (HF) }\end{array}$ & $\begin{array}{c}\text { Higher speed } \\
\text { communication and } \\
\text { farther communication } \\
\text { distance }\end{array}$ & $\begin{array}{c}\text { Communication distance } \\
\text { isn't far enough(<75CM) }\end{array}$ \\
\hline $\begin{array}{c}\text { ultrahigh frequency } \\
\text { (UHF) }\end{array}$ & $\begin{array}{c}\text { Long working } \\
\text { distance( }>1 \text { M), can } \\
\text { circumvent the object }\end{array}$ & $\begin{array}{c}\text { It has the injury to the } \\
\text { human body }\end{array}$ \\
\hline $\begin{array}{c}\text { The highest working } \\
\text { distance, highest speed } \\
\text { of communication }\end{array}$ & $\begin{array}{c}\text { Susceptibility to } \\
\text { interference, complex } \\
\text { technology }\end{array}$ \\
\hline
\end{tabular}

In the electronic toll collection system, RFID tags are affixed to the front windshield of the car, therefore, it requires compact RFID tags. At present, the $5.8 \mathrm{GHZ}$ frequency of stereoscopic RFID tags and reader are UHF products. Due to the design of the three-dimensional technology, the tags is different from the existing two-dimensional plane tags in the market, it can be directly attached to the metal, liquid, etc, and to achieve nearly $100 \%$ of the read rate. The main technical indicators of stereoscopic RFID tags and readers are shown in Table 2, hardware design of city ETC system shown in Figure IV.

TABLE II. THE MAIN TECHNICAL INDICATORS OF STEREOSCOPIC RFID TAGS AND READERS

\begin{tabular}{|c|c|}
\hline & Response time $<1.5 \mathrm{~S}$ \\
Stereoscopic & Power supply:200mW-2W \\
RFID tags & Operating temperature:-400-850 \\
\hline
\end{tabular}



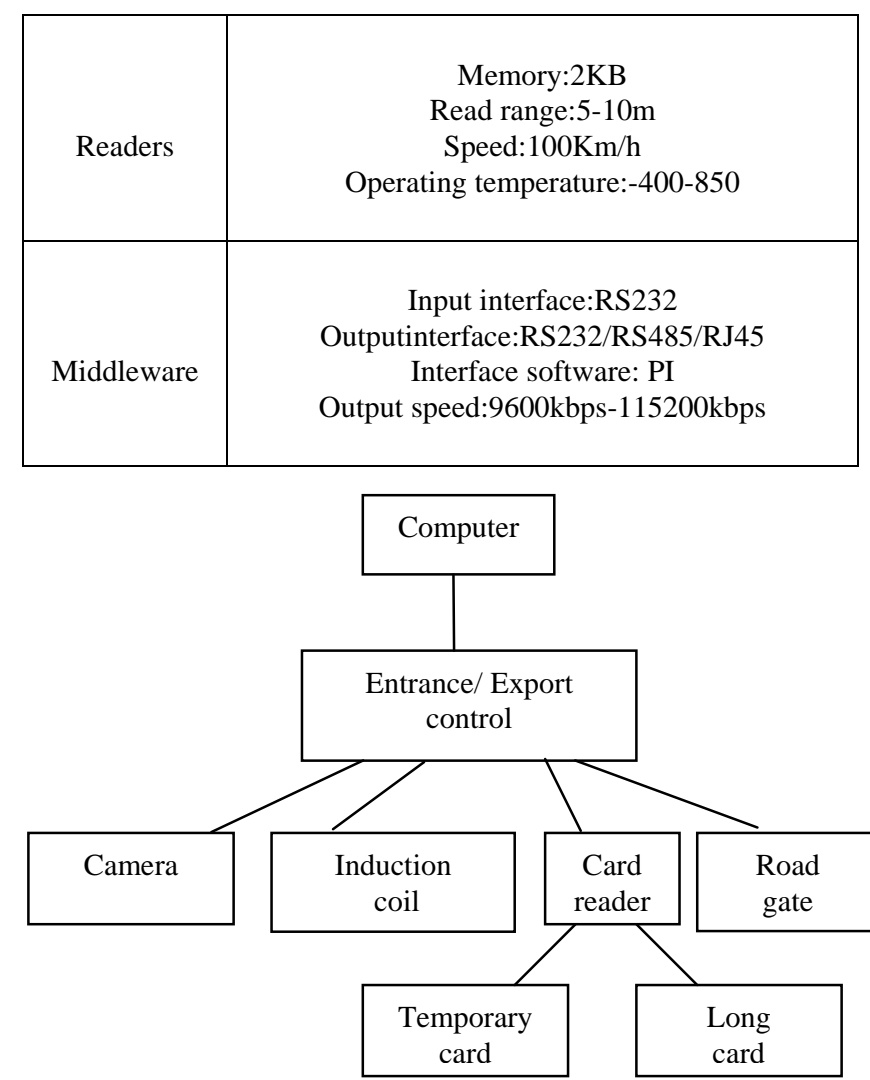

FIGURE IV. HARDWARE DESIGN OF CITY ETC SYSTEM

\section{B. ETC System Structure Based on RFID}

ETC system based on RFID mainly consists of monitoring center, access control system, RFID, video system etc, as shown in the figure III.

1) Monitoring center: Monitoring center manage the basic information of all vehicles (including time of access, image etc).it is a core part of the system. The system fully consider the data redundancy and fault-tolerant processing, then establish real-time data backup mechanism, the system can storage, statistics and query data according to customer demand.

2) Access control system: Car ban system is Information collection centers, it is responsible for the data collection work for the day-to-day and out of the vehicle, reaching safe, efficient, and orderly when vehicles enter and leave.

3) RFID: UHF RFID vehicle electronic Tags carriers of vehicle data information throughout the system, it main role is the vehicle carrying basic information transmission to the UHF RFID reader through radio frequency when vehicles come in or go out. Because the tags encoded with unique encryption of 64-bit [8], system has a higher safety factor.

4) Video systems: Through panoramic camera installed in the import and export capture the videos of the vehicles, it can be archived in the system, to facilitate the retention, can be real-time monitoring of the import and export of vehicles.
5) Others: Voice intercom system can imply the background control release by call with the monitoring center managers. The LED display is mainly used to display the state of the car park spaces and other basic information if it is required.

\section{Software Design of ETC}

When the vehicles entering the charging zone, the reader identify the car tag, By the received data of the tag, then automatically check vehicle information to determine whether the vehicle compliance requirements. If the vehicle tag does not comply with the requirements of passage, then enter illegal vehicles handler, it can take electronic pictures, post-audit fee [8]. This affects neither traffic nor cause the charging loss. If the vehicle tag complies with the requirements of passage, it can guide vehicle parking, enter the car park without stopping, capture vehicle images to store in the computer, identify the license plate, model and color information of vehicle image, These information of RFID are stored in the data processing center. When the vehicle leave the car park, capture vehicle images too, match information stored in RFID with the license plate, model and color features of vehicle image, If they are consistent then release vehicle and enter into the normal fee program to release the vehicle, otherwise alarm. If the temporary vehicle enter to the parking lot, issue a temporary electronic tags for drivers, calculate fee by models and time, drivers pay fee before leaving. The workflow is shown in Figure V.

Depending on the system design goals and requirements, the main function of the system module include: import and export control, parking guide function, vehicle identification, security monitoring, alarm functions and toll system functions etc.

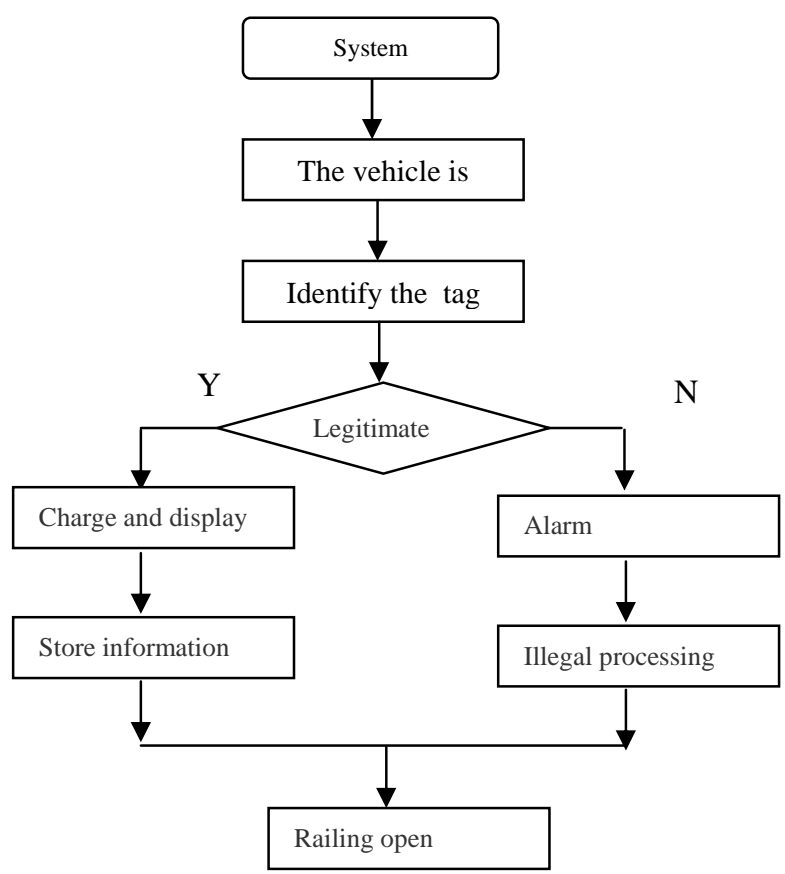

FIGURE V. WORKFLOW OF ETC SYSTEM 


\section{CONCLUSION}

In this paper, based on the specific application of the ETC system of domestic and international, according to the demand of city parking, proposed general design of city toll collection system based on RFID, included system components, the RFID principle, vehicle identification technology, anti-collision technology and system workflow etc.

\section{REFERENCES}

[1] Marci Meingst, Jennifer King, Deirdre K.Mulligan, Embedded RFID and Everyday Things: A Case Study of the Security and Privacy Risks of the U.Se-Passport, 2007 IEEE International Conference on RFID, Gaylord Texan Resort, Grapevine, TX, USA, March 26-28,2007

[2] Joseph K. Siror, Guanqun Liang, Kaifang Pang, Huanye Sheng, Dong Wang: Impact of RFID Technology on Tracking of Export Goods in Kenya. Journal of AICIT, AICIT(Journal of Convergence Information Technology) Volume 5, Number 9, October, pp 190,2010.

[3] Ying Zhang, Zhong Shen, Yilin Chang, Zhongjiang Yan, Liang Dai:A Signal Aware Movement Control Algorithm for GPS-free Ad Hoc Networks. Journal of AICIT, AICIT(Journal of Convergence Information Technology) Volume 5, Number 9, October, pp 238-245,2010.

[4] Garfinkel SL, Juels A, PappuR. RFID Privacy: an Overview of Problems and Proposed Solutions, IEEE Security \& Privacy Magazine, MayJune2005,3(3):34

[5] Transponder news-News site about radio frequency identification transponders, EAS and trolley scanning sytems.http://rapidttp.com/ transponder

[6] Yabin Li: Research on Simulation and Optimization of Transshipment Port Operation in a Power Coal Ocean Shipping Logistics System on the Basis of WITNESS. Journal of AICIT, AICIT(Journal of Convergence Information Technology) Volume 5, Number 2, April, pp 84-87, 2010.

[7] Tang Zhao-Xia, Zhang Hui, Vehicle Recognition Based on Particle Swarm Optimization and Decision Tree, Journal of AICIT, JDCTA(Journal of Digital Content Technology and its Applications) Volume 6, pp 860,2012

[8] Tang Zhao-Xia, Yang Rong-Gen, Design of city ETC system Based on the RFID and image recognition, FUJIAN Computer,2012(10):8 